Research Article

\title{
Destabilization of Immersed Dense Granular Material Submitted to Localized Fluidization: An Experimental and Numerical Study
}

\author{
Aboubacar Sidiki Dramé $\left(\mathbb{D},{ }^{1}\right.$ Li Wang $\left(\mathbb{D},{ }^{1}\right.$ and Yanping Zhang ${ }^{2}$ \\ ${ }^{1}$ School of Mechanical Engineering, University of Science and Technology Beijing, Beijing 100083, China \\ ${ }^{2}$ Central Research Institute of Building and Construction Co.Ltd., Beijing 100088, China \\ Correspondence should be addressed to Aboubacar Sidiki Dramé; magass10@xs.ustb.edu.cn and Li Wang; liwang@me.ustb.edu.cn
}

Received 4 February 2021; Revised 27 May 2021; Accepted 9 July 2021; Published 27 July 2021

Academic Editor: Jeongkwon Kim

Copyright (C) 2021 Aboubacar Sidiki Dramé et al. This is an open access article distributed under the Creative Commons Attribution License, which permits unrestricted use, distribution, and reproduction in any medium, provided the original work is properly cited.

An alternative experimental approach and a numerical analysis for the study of destabilization by localized fluidization of an immersed dense granular material are presented. To visualize the evolutions of the internal structure of the granular medium, the hydrogel beads, composed of about $99 \%$ of water and having substantially the same refraction indexes, are used as solid phase. A LED lighting system is used in place of a laser lighting system. As a result, the optical access restriction of porous structure is removed. A real economic alternative for the experimental study of fluid-grain coupling during destabilization by localized fluidization of a granular material is created. The experimental phenomenology presented in the literature is verified: the system passes successively through three different stationary regimes: static regime, fluidized cavity regime, and fluidized chimney regime. Some restrictions of using hydrogel beads as particles in the study of liquid-solid interaction are also discussed.

\section{Introduction}

In general, fluidization consists of the setting in movement of a package of particles under the effect of a fluid flow. Several techniques have been used in the past for the experimental and numerical study of the hydrodynamic coupling between the phases present in the fluidization. Among them, we can single out the experimental works, by Zoueshthiagh and Merlen [1] and Philippe and Badiane [2], and some numerical works, including for Cui et al., Marzougui et al., Jeff Ngoma et al., Montell et al. and Rigord et al. [3-7]. The study of the destabilization by localized fluidization of a granular material as well as the study of the behavior of the flows in porous media necessitated a wide use of several optical techniques: the iso-index technique and the laser-induced fluorescence (PLIF), the laser Doppler velocimetry (LDA), the particle tracking velocimetry (PTV), and the particle image velocimetry (PIV) have been extensively used [2, 8-10]. These investigation methods strongly depend on the quality of the refractive index adequacy of the solid and liquid phases [11, 12]. Many researchers have reported that, in order to visualize the evolutions of the internal structure of the granular medium without having recourse to a two-dimensional geometry, the interstitial fluid must be adapted to examples of mixtures of silicon oil, glycerol, or any other mineral oil [9, 10]. However, it is possible that the optical properties of these fluid mixtures change over time during the experiment (sensitivity of their rheological properties to temperature) [13]. Although the use of these materials has given remarkable results in the different works, the maintenance of a constant visualization through the granular materials remains the most difficult task in the experimental works. In the ultimate concern to visualize the movement of particles of the granular structure inside the porous medium, Philippe and Badiane used the iso-index technique and laser-induced 
fluorescence (PLIF) [2]. A vertical laser sheet centered on the injection zone (s) illuminates the iso-index medium thus created. A possible replacement, of the laser system, which is expensive and cumbersome and for security problems, by LED lighting, has also been the subject of several studies in the past [14-16]. Moreover, some researchers have reported that hydrogel beads are also suitable as a solid phase for visualization through granular materials. Byron and Variano [17] in their studies of the interaction of small solid bodies with a fluid medium used a solid hydrogel material which had the same index of refraction as water. Also, to measure flow velocities around solids, Weitzman et al. [18] used solid hydrogel materials. Finally, Jay et al. [19] have successfully used aqua beads to model multiphase flows. The success of these different studies took us to try the use of hydrogel beads and LED lighting for the experimental study of destabilization by localized fluidization of an immersed dense granular medium. These hydrogel beads are cross-linked materials that absorb large amounts of solvent without dissolving. These abilities make them unique materials $[20,21]$. According to the design of their matrices, the hydrogels can, by absorption, change their volume in response to stimuli, such as the temperature, the quality of the solvent, the $\mathrm{pH}$, and the electric field $[22,23]$.

An alternative experimental approach is proposed in this work. To visualize the evolutions of the internal structure of the granular medium, the hydrogels, composed of approximately $99 \%$ of water and having substantially the same refractive index as water, are used as solid phase. A threedimensional model has been developed using a numerical simulation based on the commercial software of discrete elements method (EDEM) to describe the mechanical behavior of the grain stack and a continuous model, based on the CFD ANSYS-FLUENT code, to solve the equation of fluid dynamics in confined geometry, more precisely in the pore space between grains.

\section{Experimental Setup}

The Porous medium's arrangement was done by a set of criteria (adjustment of the optical indices) with the ultimate goal of visualizing changes in the internal structure of the granular medium. We used hydrogel beads, which are denser than water when they are swollen, available commercially. The advantage of these hydrogel beads on the glass beads is that the optical access restrictions are eliminated. This also eliminates the use of complex optical techniques (need for mineral oil blends to match the refractive indices) to visualize grain motions within the granular medium. This possibility of replacing a fixed medium of transparent glass beads immersed in liquid containing mixtures of mineral oils by simple hydrogel beads in water constitutes one of the innovations of this work.

The hydrogel beads, with an initial diameter of $1 \mathrm{~mm}$, contain absorbent polymers that expand when hydrated with a solvent and can reach a diameter of 8 to $9 \mathrm{~mm}$ if the solvent is purified water. The diameter can also be of the order of 4 to $5 \mathrm{~mm}$ if the solvent is salted water since their final size is very dependent on the amount of ions in the salted water. They can contain about $99 \%$ of water if the solvent is purified water and therefore have the same index of refraction as water.

In addition, Harshani et al. [13] have shown that the diameter of these beads is kept constant for low flow rates, and that they can be used for at least three months without size and shape degradation when stored in the solvent.

A parallelepipedic cell $(1000 \times 30 \times 600 \mathrm{~mm})$ made in acrylic with transparent and good optical clarity walls was used as the main device. A series of five circular openings $(5 \mathrm{~mm}, 10 \mathrm{~mm}, 15 \mathrm{~mm}, 10 \mathrm{~mm}$, and $5 \mathrm{~mm}$ of diameter), spaced $45 \mathrm{~mm}$ each other, are drilled at the base of the cell. In order to generate a uniform, constant, and vertical flow into the cell, a single gear pump (VGS $24 \mathrm{~V}$ OEM), a flowmeter, and a water tank were used as indicated in Figure 1. To visualize the grain movements inside the granular structure, we opted for an environment enlightened by a thin light plate from a high power LED module, to create a vertical sheet centered on the zone or zones of injection, which helps to improve the quality of captured images. A fast camera, placed in front of the cell, perpendicular to the plane of the LED sheet was used to capture the illuminated zones.

At first, the cell is filled with liquid. The hydrogel beads, introduced into the cell, are arranged in bulk and packed at the bottom of the cell according to the desired stacking height. It is therefore a starting sample that is well reproducible for other initial arrangements of the experimental phase.

\section{Simulation Procedures: Methodology and Simulation Details}

A three-dimensional model, based on the coupling of two particulate methods EDEM and the CFD-FLUENT code, has been developed to simulate particle-fluid interactions in the destabilization of immersed dense granular media. EDEM, used for describing the solid particle dynamics, is a commercial software of Discrete Element Method (DEM) expanded for taking into account the bulk material simulation. The continuous model, based on the Eulerian multiphase models code in CFD-FLUENT, is used for solving the fluid dynamics equation. Figure 2 provides an overview of EDEM-FLUENT coupling process flow. The present study, which concerns the case of dense granular materials immersed, takes into account the approach of deformable bodies in contact. This approach allows for multiple particle interactions (including possible local deformation at the point of contact that is frequent in dense fluidized beds).

Particles movement is tracked individually according to Newton's motion laws:

$$
\begin{aligned}
& m_{p} \frac{d u_{p}}{d t}=F_{P}+F_{D}+F_{C}, \\
& I_{p} \frac{d \omega_{p}}{d t}=T_{p},
\end{aligned}
$$

where $m_{p}$ is the particle mass, $u_{p}$ is the particle velocity, $F_{P}$ is the particle gravity force, $F_{D}$ is the fluid-particle drag force, $F_{C}$ is the contact force, $\omega_{p}$ is the particle angular velocity, $I_{p}$ 


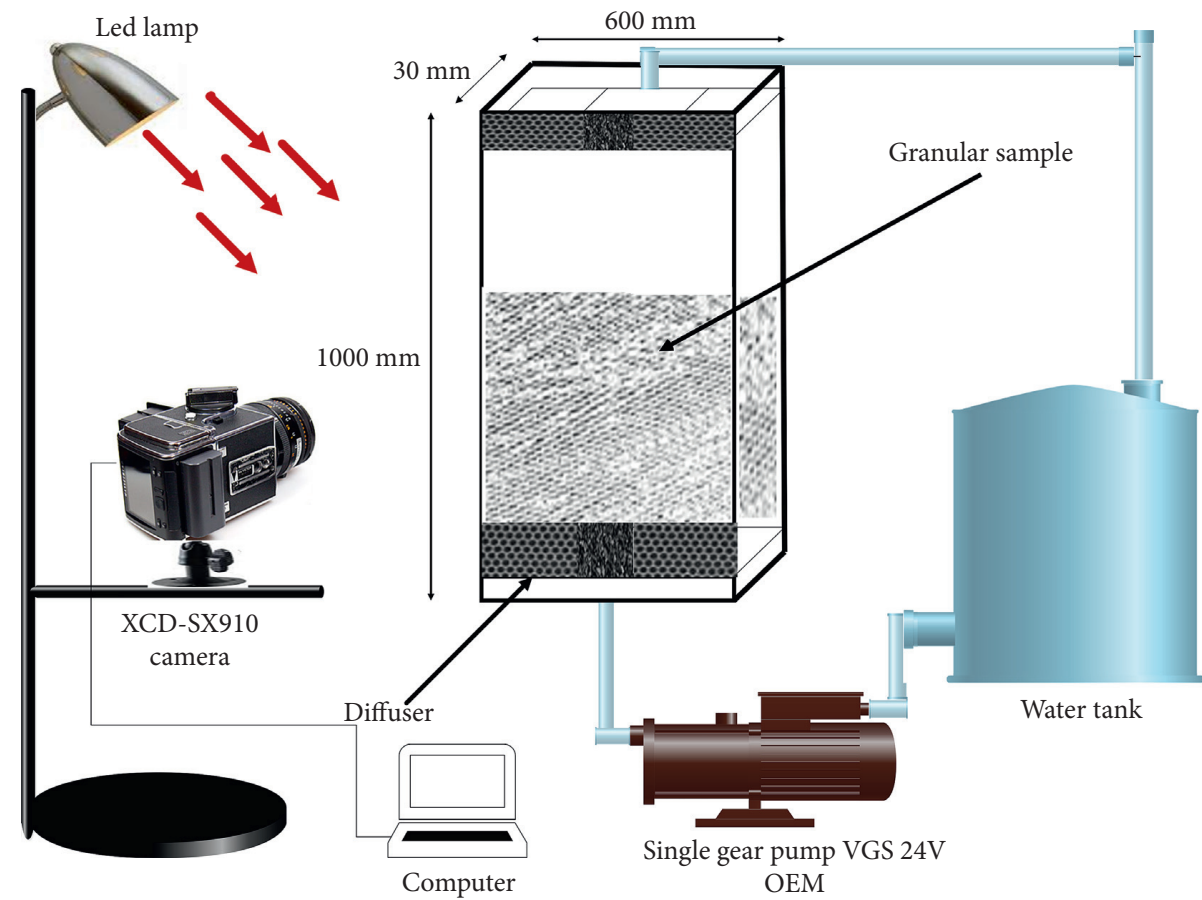

Figure 1: Illustration of the experimental device.

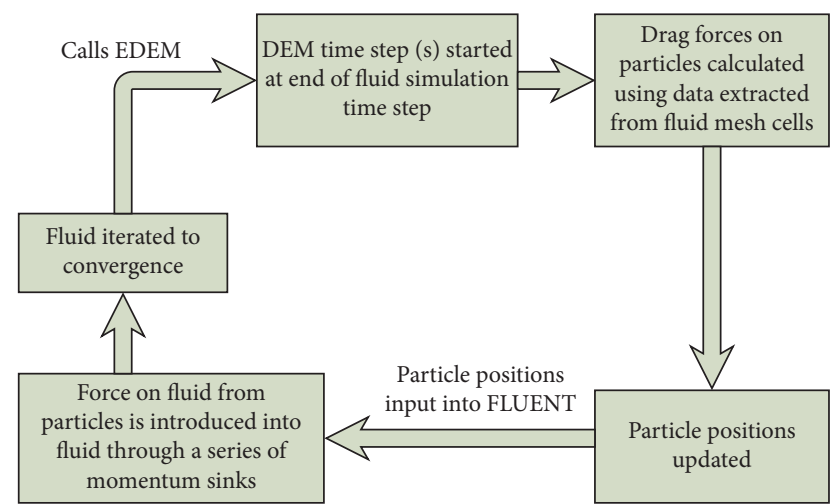

Figure 2: EDEM-FLUENT coupling Process Flow.

is the particle inertia momentum, and $T_{p}$ is the applied resulting torque on the particle.

The drag force represents the energy dissipation resulting from the effects of the fluid on the particles during the movement. It is expressed by

$$
F_{D}=\frac{\beta V_{p}}{(1-\varepsilon)}\left(u_{f}-u_{p}\right)
$$

where $V_{p}$ is the volume of particle and $\beta$ is the interphase transfer term.

The particle gravity force is expressed by

$$
F_{P}=m_{p} g=V_{p}\left(\rho_{p}-\rho_{f}\right) g .
$$

The soft sphere model, proposed by Cundal and Strack [24] and used for the first time by Tsuji et al. [25], was adopted in this study to solve all the problems of particle deformation and collision of each particle. Forces are studied as the three models (spring, dashpots, and a slider). These particle-particle and particle-wall contact forces are calculated as a function of spring constants and damping coefficients. The following enumerated equations are used to account for particle overlaps in the model of soft spheres during particle collisions:

$$
\begin{aligned}
& F_{C i}=\sum_{j}\left(f_{n, i j}+f_{t, i j}\right), \\
& T_{p i}=\sum_{j}\left(r_{i} n_{i j} * f_{t, i j}\right) .
\end{aligned}
$$

$F_{C i}$ is the net contact force acting on a particle $i$ because of its neighbors, $T_{p i}$ is the couple acting on a particle $i$ because of its neighbors, and $f_{n, i j}$ and $f_{t, i j}$ are the the normal and tangential forces on particle $i$ by particle $j$.

$$
\begin{aligned}
& f_{n, i j}=-\kappa_{n} \delta_{n, i j}-\sigma_{n} v_{n, i j}, \\
& f_{t, i j}=\kappa_{t} \delta_{t, i j}-\sigma_{t} v_{t, i j},
\end{aligned}
$$

where $\delta_{n, i j}$ and $\delta_{t, i j}$ express the normal and tangential interpenetration of the two particles $i$ and $j, \kappa_{n}$ and $\kappa_{t}$ are, respectively, the stiffness at normal and tangential contact, $\sigma_{n}$ and $\sigma_{t}$ are, respectively, the normal and tangential viscous damping coefficients, and the normal and tangential relative velocities $v_{n, i j}$ and $v_{t, i j}$ are given by the following relations:

$$
\begin{aligned}
& v_{n, i j}=\frac{d \delta_{n, i j}}{d t}=\left(\vec{v}_{i}-\vec{v}_{j}\right) \cdot \vec{n}_{i j}, \\
& v_{t, i j}=\frac{d \delta_{t, i j}}{d t}=\left(\vec{v}_{i}-\vec{v}_{j}\right)-v_{n, i j}+\left(r_{i} \omega_{i}+r_{j} \omega_{j}\right) \cdot \vec{n}_{i j} .
\end{aligned}
$$


The friction slider limits the tangential force governed by Coulomb's friction law [26].

$$
\left|f_{t, i j}\right| \leq \xi\left|f_{n, i j}\right|
$$

Water phase is modeled in CFD-FLUENT as a continuous fluid phase. The equations of continuity and momentum conservation are solved for each time step related to the fluid phase:

The continuum equation for the $q$ phase is

$$
\frac{\partial\left(\varepsilon_{q} \rho_{q}\right)}{\partial t}+\nabla \cdot\left(\varepsilon_{q} \rho_{q} \vec{u}_{q}\right)=0 .
$$

The momentum balance for the $q$ phase is

$$
\begin{aligned}
\frac{\partial\left(\varepsilon_{q} \rho_{q} \vec{u}_{q}\right)}{\partial t}+\nabla \cdot\left(\varepsilon_{q} \rho_{q} \vec{u}_{q} \vec{u}_{q}\right)= & -\nabla \cdot P+\nabla \cdot \bar{\tau}_{q}+\varepsilon_{q} \rho_{q} g \\
& -k_{s l}\left(u_{p}-u_{q}\right) .
\end{aligned}
$$

Expression $\rho_{q}$ is the $q$ phase density, $g$ is the acceleration of gravity, $\vec{u}_{p}$ and $\vec{u}_{q}$ are the phase velocities, and $\bar{\tau}_{q}$ is the $q$ phase stress-strain tensor given by

$$
\bar{\tau}_{q}=\mu_{q}\left(\frac{\partial u_{q, i}}{\partial x_{j}}+\frac{\partial u_{q, j}}{\partial x_{i}}\right)+\left(\lambda_{q}-\frac{2}{3} \mu_{q}\right) \nabla \cdot u_{q},
$$

where $\mu_{q}$ and $\lambda_{q}$ the $q$ phase's shear and bulk viscosity.

$k_{s l}$ is the interphase momentum exchange coefficient. For granular flows, momentum exchange between phases is based on the fluid-solid exchange coefficients value. In CFDANSYS Fluent, the interphase exchange coefficient models are empirically based. The Gidaspow model, which is a combination of the Wen and Yu model [27] and the Ergun equation [28], is used in this study. This model is recommended for dense fluidized beds.

$$
k_{s l}=\left\{\begin{array}{cc}
\frac{3}{4} C_{D} \frac{\varepsilon_{s} \varepsilon_{l} \rho_{l}\left|\vec{u}_{s}-\vec{u}_{l}\right|}{d_{s}} \varepsilon_{l}^{-265} ; & \varepsilon_{l}>0.8, \\
150 \frac{\varepsilon_{s}\left(1-\varepsilon_{l}\right) \mu_{l}}{\varepsilon_{l} d_{s}^{2}}+1.75 \frac{\rho_{l} \varepsilon_{l}\left|\vec{u}_{s}-\vec{u}_{l}\right|}{d_{s}} ; & \varepsilon_{l} \leq 0.8,
\end{array}\right\}
$$

where $\mu_{l}$ is the water dynamic viscosity, $d_{s}$ is the particle diameter, and $C_{D}$ is the drag force coefficient of a single particle. $C_{D}$ is expressed by

$$
C_{D}=\frac{24}{\varepsilon_{l} R_{e_{s}}}\left[1+0.15\left(\varepsilon_{l} R_{e_{s}}\right)^{0.687}\right] .
$$

Reynolds number is as follows:

$$
R_{e_{s}}=\frac{\varepsilon_{l} \rho_{l} d_{s}\left|\vec{u}_{s}-\vec{u}_{l}\right|}{\mu_{l}} .
$$

More detailed information on Gidaspow model can be found in [29].
The fluid phase is, at the start, analyzed by the commercial CFD ANSYS-FLUENT code using the solutions of the Navier-Stokes equation and the standard k-epsilon turbulence model. For a given time step, iteration of the simulation is performed in FLUENT until convergence is reached. Then, EDEM calculates body forces acting on each particle (gravity, fluid drag, electrostatic) using data extracted from fluid mesh cells and eventually determines updated particle position, acceleration, and velocity. These different coordinates of the particles thus updated are transferred to the CFD solver ANSYS-FLUENT and the forces exerted on the fluid by the particles are introduced into the fluid through a series of momentum source terms.

The simulations were carried out on a granular of silicon-si assembly which constituted the discrete phase provided by EDEM whereas the liquid water of kinematic viscosity $v_{f}=2.010-6 \mathrm{~m}^{2} \mathrm{~s}^{-1}$ provided by FLUENT constituted the continuous phase. The laminar flow option and the double precision mode were selected in CFD-FLUENT. The particles of spherical form had a density of $2500 \mathrm{~kg} \mathrm{~m}^{-3}$ and an average size of $1.610^{-3} \mathrm{~m}$ while the density of the water is $1000 \mathrm{~kg} \mathrm{~m}^{-3}$. In order to certify the experimental observations, a parallelepipedic cell of $600 \times 30 \times 1000 \mathrm{~mm}$ constituted the computation domain for the simulations work. Figure 3 shows the computing domain meshed using the Integrated Computer Engineering and Manufacturing (ICEM) code and the simulation domain with EDEM particles. Sides of the mesh model were specified as wall boundary conditions, holes in the bottom were (Figure 3(c)) specified as velocity inlet according to corresponding simulation phase, and the upper surface was specified as an outflow boundary. The selection of the turbulence model affects the analysis because each model uses a different set of boundary conditions. The Reynolds-Averaged Naiver-Stokes (RANS) simulates turbulence in flow as a result of averaging the steady-state and dynamic flow variables. We adopted this model because of its simplicity and ease of understanding. Accurate fine grid resolution is essential to detect the smallest vortex formation in the turbulent flow; therefore high computing capacity is desired. And it is shown that, with generally more fine grids, all iterative solution methods converge with a good accuracy. The computation may require significantly more time [30]. Moreover, to capture the particles behavior accurately, a smaller integration time step is also essential. The computational domain consists of a total of 2979507 finer grids with a total of 594217 nodes. In addition, a time step of $10^{-4} \mathrm{~s}$ is chosen in this work, which is supposed to be certainly small enough to produce reasonably accurate results. The validation of this numerical model is also based on the visual observations of dynamic process during the experimental work. Moreover, CFD-DEM two-way coupling have been more used in recent years to deal with the gas-particle coupling in two-phase flow systems with accurate results [31].

Furthermore, the direction of acceleration of gravity $\left(g=9.81 \mathrm{~m} . \mathrm{s}^{-2}\right)$ was evaluated as being opposite to the direction of flux entering the domain entrance. Then, we imposed a flow velocity $U$ greater than the fluidization 


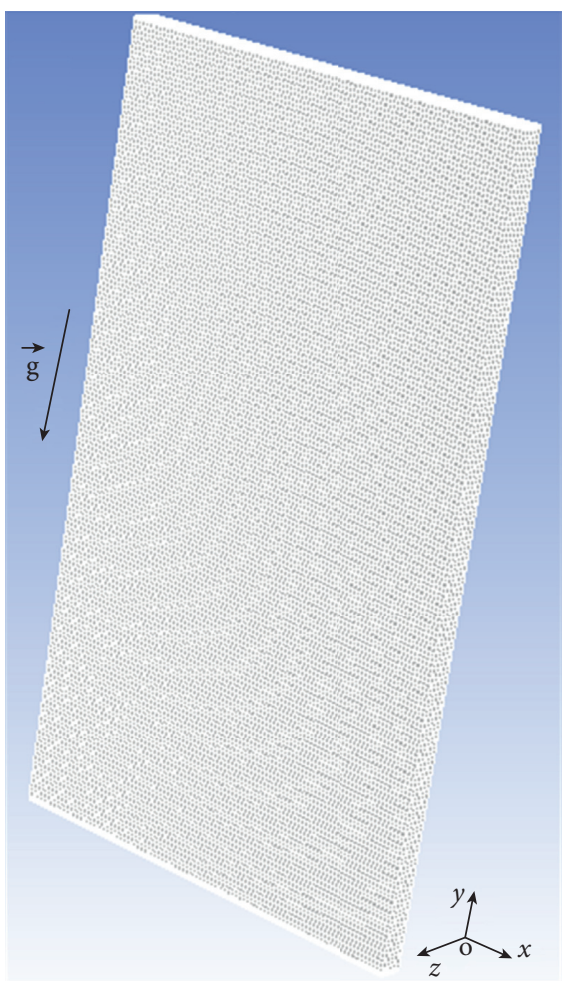

(a)

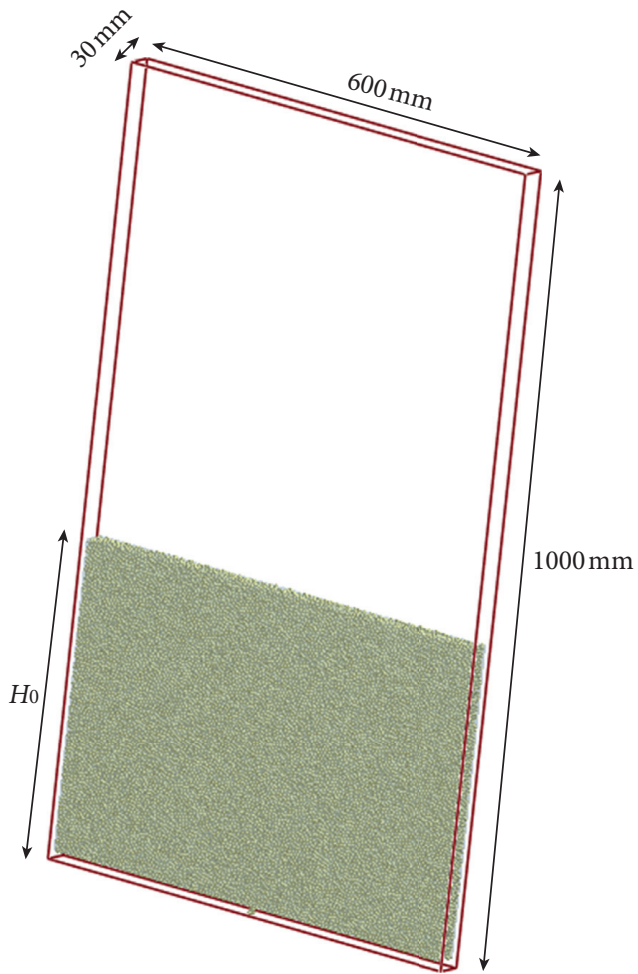

(b)

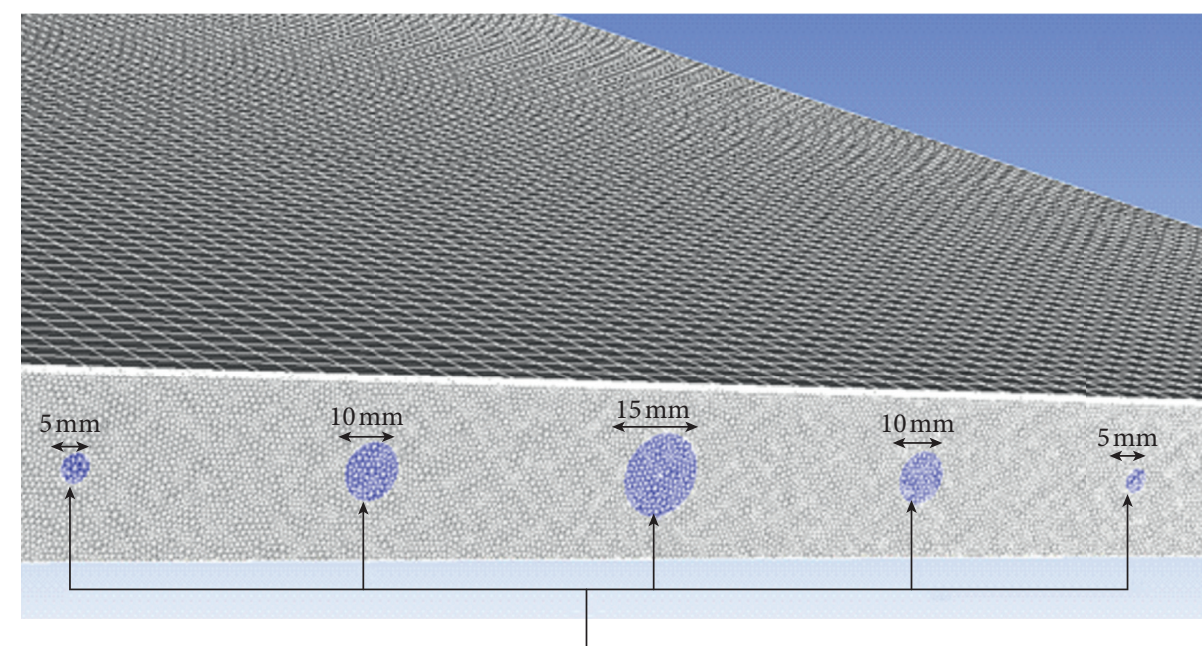

Five (05) openings, which have served as velocity inlet

(c)

FIgURE 3: (a) ICEM grid, (b) simulation domain with EDEM particles, and (c) inlet holes at the base of the box.

threshold, at the base of the granular layer, and kept constant during the duration of the simulation.

\section{Results and Discussion}

In this work, the collapse or partial rupture of a civil engineering structure in the presence of water infiltration by fluidization is studied in the laboratory through a model based on a granular stack subjected to an ascending liquid flow. Also, a three-dimensional model, based on the coupling of two particulate methods EDEM and the CFDFLUENT code, has been developed to realistically report the predominant physical mechanisms involved in our experimental studies. This study of the fluid-grain interaction is motivated by the fact that the resulting phenomena are sometimes at the origin of the appearance of hydromechanical instabilities. The fluid exerts aerodynamic or hydrodynamic forces on the grain interface, which can then move or deform. This displacement or deformation of the grains also affects the flow field locally. A better 


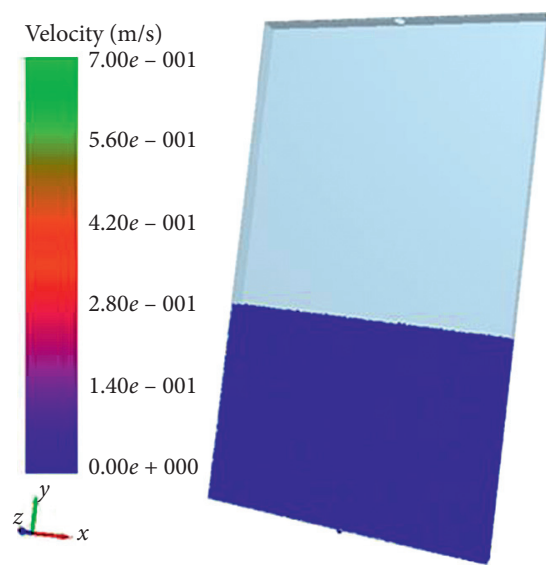

(a)

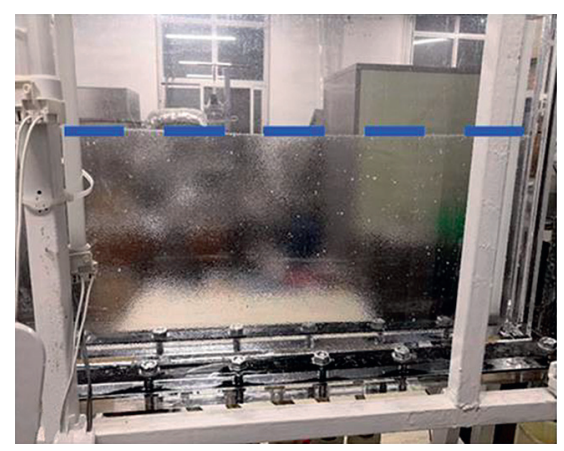

(b)

FIgURE 4: Image illustration of the static steady regime: (a) numerical observation; (b) experimental observation.

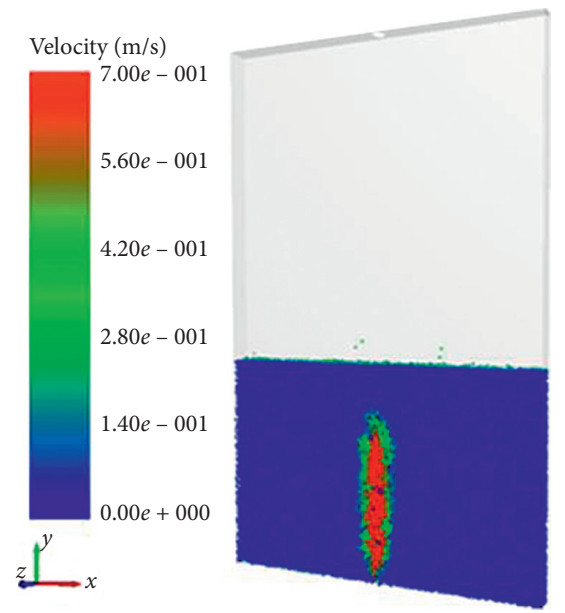

(a)

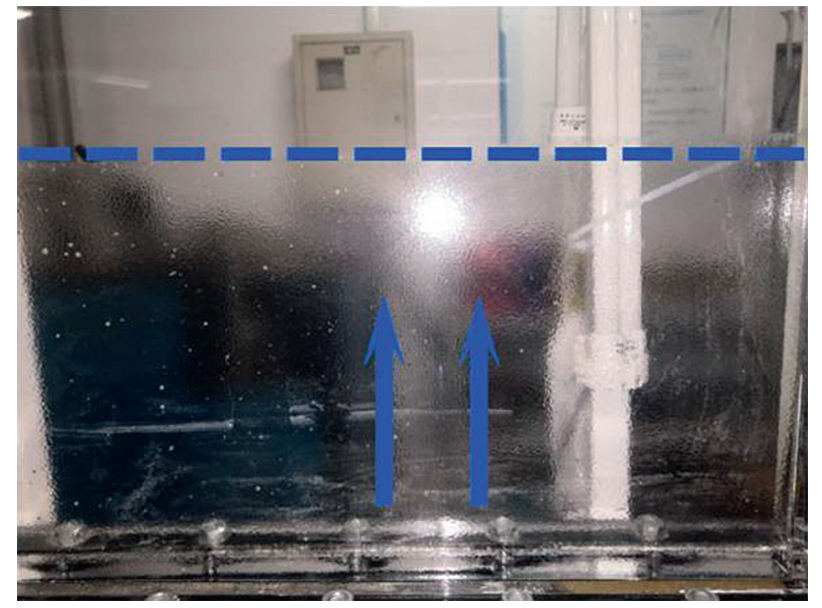

(b)

FIGURE 5: Image illustration of the fluidized cavity regime: (a) numerical observation; (b) experimental observation.

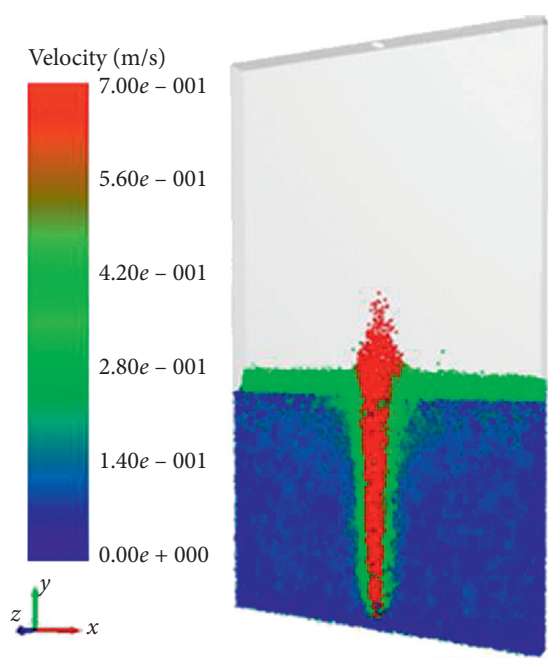

(a)

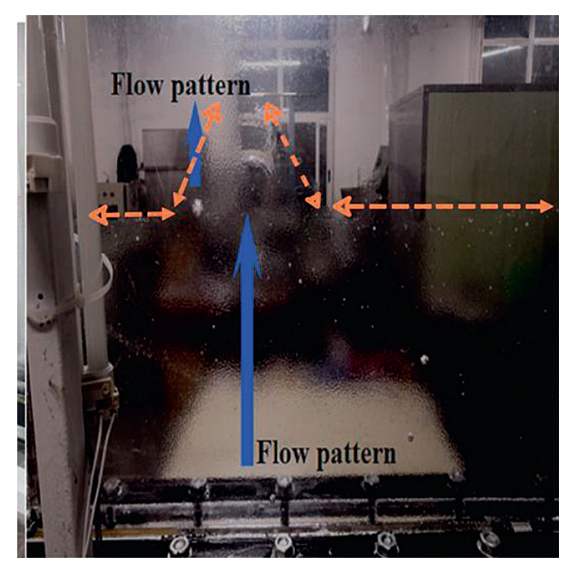

(b)

FIGURE 6: Image illustration of the fluidized chimney regime: (a) numerical observation; (b) experimental observation. 
understanding of fluid-grain coupling and its role in the development of hydro mechanical instabilities can help to respond to a concrete problem of the safety of hydraulic structures, in which the fluidization located at the foot appears as an initiating mechanism of regressive erosion.

We looked at the case of localized fluidization of an immersed grain layer with the single nozzle located at the center of the cell. For different flow rates through an injection diameter of $d_{\mathrm{i}}=15 \mathrm{~mm}$, we observe the growth of the hydromechanical instability which is created at the level of the injection nozzle and propagates within the granular structure up to the surface of the bed.

When the injection speed $\mathrm{U}$ is regularly increased, the phenomenology demonstrated in the experiments $[1,2]$ is perfectly highlighted. The bed is described successively by three different regimes:

(i) The static regime, which corresponds to the low injection velocities; there is no deformation of the granular stack or significant movement of grains all along the bed. The stack behaves like a fixed porous medium.

(ii) The regime of the fluidized cavity corresponding to the sufficient injection velocities: we observe not only a significant grains movement just above the injection nozzle but also a local deformation of the stack at the surface of the injection zone.

(iii) The fluidized chimney regime corresponding to higher injection velocities: we observe a local fluidization throughout the thickness of the bed and the presence of a vertical chimney above the injection zone.

Indeed, an imbalance of the contact forces induced by the flow on the grains causes the destabilization phenomenon. When the induced forces are more significant than the apparent weight of the granular stack, there can be fluidization with large relative movements of grains in interior and a more or less regular expansion of the whole material. The lower surface was gradually destabilized thus releasing a shower of grains that gradually fed the fluidized zone. The size of the ascending grain layer in solid translation motion decreased regularly while the fluidized layer increased. There is also fracturing of the bed's injection zone surface. In fact, the internal structure of the granular stack is reorganized by retroacting on the interstitial flow thus leading to the opening of a preferential path of low hydraulic resistance for the liquid flow and the modification of the dynamic behavior of the grains.

More information about the physical phenomenon involved in the three cited regimes can be found in [1].

To report more on the situation in which the stresses exerted by a liquid flow in a submerged granular medium are sufficient to put in motion certain grains, simulation works based on the coupling of two particular methods (EDEM and CFD ANSYS FLUENT) have been carried out. The same dynamic of growth of the fluidized zone in an initially static immersed granular stack was found with the simulations work. Figures 4-6 illustrate the three different stationary regimes observed during the experimental and the numerical works for a stack of initial height $H_{0}=400 \mathrm{~mm}$ and with an injection diameter $d_{\mathrm{i}}=15$ : the static regime, the fluidized cavity regime, and the fluidized chimney regime. Moreover, by observing the behavior of the bed during the experimental phase, it is clear that the numerical observations show a good agreement with the experimental observations. However, other more in-depth studies devoted to these numerical and experimental observations are in progress and will be the subject of further submissions in the near future.

\section{Conclusion}

In natural environments, it is common to observe particular cases of vertical localized fluidization, a phenomenon which manifests itself as a hydraulic flow of infiltration into the foundations of the structure in Earth and can cause an overall lifting and possibly a hydraulic fracturing. The present work consists of experimental and numerical study of the destabilization by localized fluidization of an immersed dense granular material through the development of a new PIV approach able to correctly reproduce the interactions between fluid phase and solid phase. The aim was to force an ascending fluid through a bed of particles, which remain fixed until the flow reaches a certain flow rate, called the minimum flow of fluidization. Beyond this flow, when the drag force of the fluid completely offsets the effective weight of the particles, they are then fluidized. It seeks to understand the concrete problem of the safety of hydraulic structures, in which the localized fluidization at the foot appears as a mechanism initiating regressive erosion, one of the internal erosion modes. Restriction of the optical access to the porous structure was removed using hydrogel beads as solid particles and water as the fluid having substantially the same refractive index. A LED lighting system was used instead of a laser lighting system, thus avoiding a lot of risk of manipulation. A real economic alternative for the experimental study of fluid-grain coupling during destabilization by localized fluidization of a granular material was created.

The results of this study of destabilization by fluidization of an immersed grain layer by a variable size basal injection showed the observation of experimental phenomenology presented in literature (the fluidized bed passed successively by the static regime, the fluidized cavity regime, and the fluidized chimney regime). The committed effort must continue; a low-cost PIV system and hydrogel beads as a solid phase, reducing health and safety requirements at work, may well replace an expensive lazer lighting system and a mixture of liquids. The use of such type of inexpensive PIV systems in the study of the fluidization destabilization of a granular material may be tainted by certain difficulties such as the presence at the market of hydrogel beads which have densities similar to that of water when they are swollen (possibility of floating of balls in the water). This is avoidable by communicating to the seller all the characteristics of the desired hydrogel beads. The hydrogel beads should also be washed or changed after a series of tests for good visibility 
through the porous medium. We must also take advantage of the information provided to better understand qualitatively, but especially quantitatively, the mechanisms leading to the destabilization of grains. In perspective, more detailed analysis should be carried out on the mechanisms leading to the destabilization of grains and the mode of expansion of the instabilities according to the hydrodynamic regime.

\section{Data Availability}

The data used to support the findings of the article are available on reasonable request to the corresponding author.

\section{Conflicts of Interest}

The authors declare that they have no conflicts of interest.

\section{Acknowledgments}

The National Key R\&D Program of China (Grant no. 2016YFB0601101) and the Open Fund Project for State Key Laboratory of Iron and Steel Industry Environmental Protection (No. 2016YZC04) supported this work.

\section{References}

[1] F. Zoueshtiagh and A. Merlen, "Effect of a vertically flowing water jet underneath a granular bed," Physical Review E, vol. 75, no. 5, pp. 1-12, 2007.

[2] P. Philippe and M. Badiane, "Localized fluidization in a granular medium," American Physical Society, vol. 42206, pp. 31-33, 2013.

[3] X. Cui, J. Li, A. Chan, and D. Chapman, "Coupled DEM-LBM simulation of internal fluidisation induced by a leaking pipe," Powder Technology, vol. 254, pp. 299-306, 2014.

[4] D. Marzougui, B. Chareyre, and J. Chauchat, "Microscopic origins of shear stress in dense fluid-grain mixtures," Granular Matter, vol. 17, no. 3, pp. 297-309, 2015.

[5] E. P. Montell, M. Toraldo, B. Chareyre, and L. Sibille, "Localized fluidization in granular materials: theoretical and numerical study," Physical Review E, vol. 94, pp. 1-14, 2016.

[6] P. Rigord, A. Guarino, V. Vidal, and J.-C. Géminard, "Localized instability of a granular layer submitted to an ascending liquid flow," Granular Matter, vol. 7, no. 4, pp. 191-197, 2005.

[7] J. Ngoma, S. Bonelli, P. Philippe, J. Delenne, and F. Radjai, "Interaction between two localized fluidization cavities in a granular media: experiments and numerical simulation," in Modeling Granular Media across Scales-MGMAS2014, Article ID 42206, Montpellier, France, 2014.

[8] M. Giese, K. Rottschafer, and D. Vortmeyer, "Measured and modeled superficial flow profiles in packed beds with liquid flow," AIChE Journal, vol. 44, no. 2, 1998.

[9] A. Y. L. Huang, M. Y. F. Huang, H. Capart, and R.-H. Chen, "Optical measurements of pore geometry and fluid velocity in a bed of irregularly packed spheres," Experiments in Fluids, vol. 45, no. 2, pp. 309-321, 2008.

[10] V. A. Patil and J. A. Liburdy, "Flow characterization using PIV measurements in a low aspect ratio randomly packed porous bed," Experiments in Fluids, vol. 54, 2013.

[11] R. Budwig, "Refractive index matching methods for liquid flow investigations," Experiments in Fluids, vol. 17, no. 5, pp. 350-355, 1994.
[12] J. A. Dijksman, F. Rietz, K. A. Lrincz, M. Van Hecke, W. Losert, and M. Van Hecke, "Invited Article: refractive index matched scanning of dense granular materials," Review of Scientific Instruments, vol. 83, no. 1, 2013.

[13] H. M. D. Harshani, S. A. Galindo-Torres, A. Scheuermann, and H. B. Muhlhaus, "Experimental study of porous media flow using hydro-gel beads and LED based PIV," Measurement Science and Technology, vol. 28, no. 1, p. 15902, 2017.

[14] N. A. Buchmann, C. E. Willert, and J. Soria, "Pulsed, highpower LED illumination for tomographic particle image velocimetry," Experiments in Fluids, vol. 53, no. 5, pp. 1545-1560, 2012.

[15] H. M. D. Harshani and A. Scheuermann, "Particle image velocimetry with high power light emitting diodes for flow through porous medium," Computational Methods in Multiphase Flow VIII, vol. 89, pp. 501-511, 2015.

[16] C. Olivier and K. Kyung Chun, "Miniature particle image velocimetry system with LED in-line illumination," Measurement Science and Technology, vol. 13, no. 7, pp. 1006-1013, 2002.

[17] M. L. Byron and E. A. Variano, "Refractive-index-matched hydrogel materials for measuring flow-structure interactions," Experiments in Fluids, vol. 54, pp. 1-17, 2013.

[18] J. S. Weitzman, L. C. Samuel, A. E. Craig, R. B. Zeller, S. G. Monismith, and J. R. Koseff, "On the use of refractive index - matched hydrogel for fluid velocity measurement within and around geometrically complex solid obstructions," Experiments in Fluids, vol. 55, 2014.

[19] L. H. Jay, T. Kazunori, I. Magued, and Y. Sung-ho, “A transparent water-based polymer for simulating multiphase flow," Geotechnical Testing Journal, vol. 33, no. 1, Article ID 102375, 2010.

[20] T. Tanaka, Gels, Scientific. Scientific American: Scientific American, 1981.

[21] M. Shibayama and T. Tanaka, "Volume phase transition and related phenomena of polymer gels," Advances in Polymer Science, vol. 109, 1993.

[22] K. Dusek and D. Patterson, "Transition in swollen polymer networks induced by intramolecular condensation," Journal of Polymer Science, vol. 1216, pp. 1209-1216, 1968.

[23] T. Tanaka, "Collapse of gels and the critical endpoint," Physical Review Letters, vol. 40, no. 12, pp. 820-823, 1978.

[24] P. A. Cundall and O. D. L. Strack, "A discrete numerical model for granular assemblies," Géotechnique, vol. 29, no. 1, pp. 47-65, 1979.

[25] Y. Tsuji, T. Kawaguchi, and T. Tanaka, "Discrete particle simulation of two-dimensional fluidized bed," Powder Technology, vol. 77, no. 1, pp. 79-87, 1993.

[26] A. Di Renzo and F. P. Di Maio, "Comparison of contact-force models for the simulation of collisions in DEM-based granular flow codes," Chemical Engineering Science, vol. 59, no. 3, pp. 525-541, 2004.

[27] C. Y. Wen and Y. H. Yu, "Employment information," Chemical \& Engineering News Archive, vol. 44, no. 19, pp. 100-111, 1966.

[28] S. Ergun, "Fluid flow through packed columns.pdf," Chemical Engineering Progress, vol. 48, no. 2, pp. 89-94, 1952.

[29] D. Gidaspow, R. Bezburuah, and J. Ding, "Hydrodynamics of circulating fluidized beds, kinetic theory approach," Proceedings of the 7th Engineering Foundation Conference on Fluidization, vol. 7, pp. 75-82, 1992.

[30] M. A. El-Emam, L. Zhou, W. Shi et al., "Theories and applications of CFD-DEM coupling approach for granular flow: 
a review," in Archives of Computational Methods in Engineering, Springer, Berlin, Germany, 2021.

[31] L. Zhou, C. Han, L. Bai, W. Li, M. A. El-Emam, and W. Shi, "CFD-DEM bidirectional coupling simulation and experimental investigation of particle ejections and energy conversion in a spouted bed," Energy, vol. 211, Article ID 118672, 2020 . 